\title{
Platelets count and platelets indices; mean platelet volume and plateletcrit in pediatric chronic lung disease
}

\author{
Iman H. Draz ${ }^{1 *} \mathbb{D}$, Iman A. Shaheen ${ }^{2}$ and Eman A. Youssef ${ }^{2}$
}

\begin{abstract}
Background: The lungs contribute in the final maturation of the circulating platelets, and some studies suggested that chronic lung disease (CLD) could impair circulating platelets maturation or count. Our aim was to study the validity of platelet count (PLT), mean platelet volume (MPV), and plateletcrit (PCT) as simple tools to assess chronic lung disease severity or predict its course.

Results: In 39 pediatric patients with CLD, we showed a significant rise in PLTs and PCT and a significant lower MPV when compared to normal controls ( $P$ values $0.003,0.010,0.004$, respectively). Also, a significant positive correlation was detected between the $C$ reactive protein (CRP) level and both the PLTs and PCT $(r$ 0.327, 0.363 respectively) in addition to a significant reduction in MPV level in relation to the age of disease onset ( $P$ value 0.031).

Conclusion: This study disagrees with the postulation that chronic lung disease could lead to the reduction of circulating platelets and emphasizes the role of increased platelets count and decreased MPV in reflecting the inflammatory condition underlying the chronic lung disease in the pediatric age group.
\end{abstract}

Keywords: Chronic lung disease, Pediatric, Platelets count, Mean platelet volume, Plateletcrit

\section{Background}

Chronic lung diseases in children such as bronchiectasis and interstitial lung disease represent a major public health problem with limited therapeutic options [1]. The disease severity is highly variable according to the underlying condition but unfortunately, children with CLD have high overall morbidity and mortality [2]. Therefore, it is very important to find a non-invasive blood biomarker that could help in the diagnosis and predict the prognosis of ILD, it would be of more value in areas with limited resources to allow easier and earlier detection of more vulnerable patients [3].

The murine model of chronic obstructive pulmonary disease (COPD) has shown that lungs may serve as a reservoir for megakaryocytes and hematopoietic progenitor cells, where almost half of the platelets may be produced [4]. This finding sheds new light on thrombopoiesis in

\footnotetext{
* Correspondence: imane.draz@cu.edu.eg; imane.draz@yahoo.com

${ }^{1}$ Pediatrics, Pediatric Pulmonology, Faculty of Medicine, Cairo University, Kasr

Alainy, Street, Cairo Post Code: 11562, Egypt

Full list of author information is available at the end of the article
}

mammals and raises a question of whether pulmonary platelet production takes place in the human lungs as well. If it were so, chronic pulmonary diseases that adversely affect lung tissue could affect the platelet count [5].

It was suggested that decreased mean platelet volume (MPV), one of the parameters routinely generated by blood counters, might indicate increased systemic inflammation during COPD exacerbation and that MPV could be a useful tool in the assessment of the inflammatory response [6].

Plateletcrit (PCT) provides more comprehensive data about total platelet mass because it reflects both the MPV and platelet count (PLT), where PCT is calculated by multiplying the PLT by the MPV $/ 10^{7}$ [7]. Platelet activation in infectious and allergic lung diseases has been well documented, platelets have been found extravascular in the lungs of patients with asthma, and in animal models of allergic lung inflammation. Previous studies demonstrated that blood leaving the lungs contained more platelets and fewer megakaryocytes than blood entering the lungs [8]. 
The aim of this study was to study changes in parameters of platelets in pediatric patients with CLD. The platelet count, MPV and PCT all represent laboratory parameters that are easy to obtain and readily available in most treatment facilities, if such elements could be applied as tools for assessment of the disease severity or to predict upcoming exacerbations or early prediction of a treatment response it would save much effort and cost.

\section{Methods}

Between September 2018 and March 2019, 121 patients with chronic lung disease presented to the pulmonary unit at the pediatric teaching hospital for follow-up. Of these, 39 subjects were recruited in this cross-sectional case-control study. Chronic respiratory diseases were defined as the presence of respiratory symptoms for 3 months or more in each of 2 consecutive years in a patient in whom other causes of such respiratory symptoms have been excluded.

The patients group constituted 23 (58.9\%) males and $16(41.0 \%)$ females, their ages ranged from 2 to 13 years with a mean of $6.9 \pm 3.41$ years, they were then compared with a group of 40 healthy control subjects with matched age and sex distribution randomly chosen from the general clinic, the normal control group constituted 18 (45\%) males and 22 (55\%) females, their ages ranged from 1.5 to 13 years with a mean of $6.1 \pm 3.05$ years. Patients were then stratified into two groups: interstitial lung disease $(n=20)$ and non-cystic fibrosis (CF) bronchiectasis $(n=19)$ based on high-resolution computed topography (HRCT) findings.

Exclusion criteria included any associated disorders that may affect the platelet count as kidney failure, autoimmune diseases, diabetes, malignancies, chronic liver disease, and patients with prior treatment with anticoagulant. Both groups were enrolled in the study after obtaining informed consent from the patients' legal guardians and the approval of the institutional ethical committee. Confidentiality of the data was guaranteed in handling the database according to the revised Helsinki Declaration of biomedical ethics [9].

Blood count was performed using an automated hematology analyzer "XN-1000 Sysmex" which measures blood count parameters including total leucocytic count (TLC), red blood cells (RBCs) count, RBCs mean corpuscular volume $(\mathrm{MCV})$, platelet count, and mean platelet volume (MPV) by direct current detection method, blood film scanning of individual cases was done to exclude any analytical errors. Plateletcrit was calculated by multiplying MPV and platelet count to assess the functioning platelet mass. Erythrocyte sedimentation rate (ESR) was measured using the Westergren tube method for both groups, Creactive protein (CRP) was quantitatively measured by the automated Beckman coulter AU 480 chemistry analyzer with cut off value $5 \mathrm{mg} / \mathrm{L}$.

\section{Data analysis}

Data were analyzed using the statistical package for the Social Sciences (SPSS) version 25 (IBM Corp., Armonk, NY, USA). Data was summarized using mean, standard deviation, median, minimum, and maximum in quantitative data and using frequency (count) and relative frequency (percentage) for categorical data. Comparisons between quantitative variables were done using the nonparametric Kruskal-Wallis and Mann-Whitney tests [10]. Correlations between quantitative variables were done using the Spearman correlation coefficient [11]. $P$ values less than 0.05 were considered statistically significant.

\section{Results}

The current study revealed that pediatric patients with CLD had significantly higher platelet count when compared to the normal control group ( $P$ values 0.003 ), we also showed a significant decrease in the MPV among the patients group when compared to the normal control group ( $P$ values 0.004$)$, the plateletcrit was significantly higher when compared to the normal group ( $P$ value 0.010). ESR and CRP were significantly higher in the diseased group ( $P$ values 0.00 ) (Table 1$)$. When the patients' group was further divided into interstitial lung disease and non-CF bronchiectasis then compared to the normal group, the MPV was significantly lower in both patients' subgroups ( $P$ values $0.007,0.048$ respectively); however, there was no statistically significant difference between the 2 patients' subgroups regarding the MPV nor the PCT. (Table 2).

Platelet count, MPV, and plateletcrit where studied among the CLD group and compared to different parameters including age, age of onset, oxygen saturation, hemoglobin concentration, TLC, ESR, CRP, presence of fever, and changing pattern of chronic cough, sputum, or dyspnea. On comparing the platelet parameters (platelet count, MPV, and PCT) against other parameters, significant negative correlation existed between the MPV and age $(P$ value $0.015, r-0.388)$, also significant negative correlation existed between the MPV and age of start of illness $(P$ value $0.031, r-0.346)$. Significant negative correlation existed between the platelet count and hemoglobin concentration ( $P$ value $0.032, r-0.343$ ). Significant positive correlation existed between the platelet count and PCT in relation to the CRP ( $P$ value $0.042, r 0.327$ and $P$ value $0.023, r 0.363$ respectively) (Tables 3 and 4 )

There was no statistically significant difference on correlating the platelet parameters (platelet count, MPV and PCT) among patients who presented with fever, changing pattern of chronic cough, sputum, or dyspnea and those who did not present with those symptoms, 
Table 1 Comparison between cases and controls regarding CBC findings and other labs

\begin{tabular}{|c|c|c|c|}
\hline Parameters & Cases $(n=39)$ & Controls $(n=40)$ & $P$ value \\
\hline \multicolumn{4}{|l|}{$H B$} \\
\hline Range & $8.5-15.2$ & $8.6-14.8$ & \\
\hline Mean \pm SD & $11.6 \pm 1.3$ & $11.8 \pm 1.5$ & 0.518 \\
\hline \multicolumn{4}{|l|}{ TLC } \\
\hline Range & $4.6-24$ & $3.9-14.2$ & \\
\hline Mean \pm SD & $11.2 \pm 4.9$ & $7.9 \pm 2.8$ & 0.001 \\
\hline \multicolumn{4}{|l|}{ PLTS } \\
\hline Range & $154-819$ & $123-438$ & \\
\hline Mean \pm SD & $394.8 \pm 175.6$ & $278.2 \pm 85.8$ & 0.003 \\
\hline \multicolumn{4}{|l|}{ MPV } \\
\hline Range & $7.60-10$ & $8.10-10.0$ & \\
\hline Mean \pm SD & $8.68 \pm 0.57$ & $9.07 \pm 0.54$ & 0.004 \\
\hline \multicolumn{4}{|l|}{ PCT } \\
\hline Range & $0.122-2.20$ & $0.123-3.227$ & \\
\hline Mean \pm SD & $0.396 \pm 0.339$ & $0.324 \pm 0.477$ & 0.010 \\
\hline \multicolumn{4}{|l|}{$\mathrm{HCT}$} \\
\hline Range & $25.5-45.6$ & $29-43$ & \\
\hline Mean \pm SD & $34.8 \pm 4$ & $36.6 \pm 3.4$ & 0.031 \\
\hline \multicolumn{4}{|l|}{ ESR } \\
\hline Range & $12-70$ & $2-22$ & \\
\hline Mean \pm SD & $38.3 \pm 16.3$ & $10.8 \pm 4.4$ & 0.000 \\
\hline \multicolumn{4}{|l|}{ CRP } \\
\hline Elevated & $29(72.5 \%)$ & $0(0)$ & 0.000 \\
\hline Normal values & $11(27.5 \%)$ & $40(100)$ & \\
\hline
\end{tabular}

$P P$ value, $H B$ hemoglobin, $T L C$ total leucocyte count, $P L T$ platelets, MPV mean platelets volume, $P C T$ plateletcrit, $H C T$ hematocrit, ESR erythrocyte sedimentation rate CRP C-reactive protein

Italic is significant

also no significant correlation existed between the previously mentioned platelet parameters and degree of oxygen saturation among the patients group.

\section{Discussion}

Recent studies on murine models have shown that maturation of platelets takes place, to some extent in the lungs, this raises the possibility that chronic lung diseases could affect platelet maturation or count [4]. Chronic lung

Table $2 P$ value for the comparison between interstitial lung disease group, non-CF bronchiectasis group, and normal control group

\begin{tabular}{lll}
\hline & MPV & Pltcrit \\
\hline Interstitial lung disease vs non-CF bronchiectasis & 0.566 & 0.720 \\
Interstitial lung disease vs controls & 0.007 & 0.021 \\
Non-CF bronchiectasis vs controls & 0.048 & 0.063 \\
\hline
\end{tabular}

MPV mean platelets volume, $P C T$ plateletcrit disease carries many challenges for the patients and their caregivers, such as having an unpredictable clinical course, absence of a definitive treatment, restriction of activities whether due to the disease or the medications as well as later psychosocial effects [2]. Our study found a significant rise in platelet count in the patients group when compared against the normal control group, this comes in agreement with the study by Maclay et al. [12] which may be attributed to hypoxemia, underlying inflammation, and oxidative stress, although it disagrees with results obtained by Skoczyński et al. [13] both studies however were carried on adult COPD patients.

In agreement with our current study, the number of platelets in the COPD patients was inversely associated with the hemoglobin level [13]. The cause of anemia in COPD is related to certain pro-inflammatory markers, at least a component of the anemia is attributable to inflammation (anemia of chronic disease) [14].

CLD is associated with low-grade systemic inflammation, activation of circulating inflammatory cells, and increased levels of inflammatory cytokines. This is postulated to explain the significant rise in both CRP and ESR among the patients group when compared to the normal control. We also found a significant reduction in the MPV among patients, and when the patients' group was subdivided into Interstitial lung disease and non-CF bronchiectasis both subgroups still had a significantly lower MPV when compared to the normal control but not when compared to each other. Low levels of MPV are associated with highgrade inflammatory diseases and reverse in the course of anti-inflammatory therapy [15].

Mean platelet volume (MPV) is one of the parameters routinely generated by blood counters; it reflects the platelet production rate and stimulation. An inverse correlation between MPV and disease activity was demonstrated in inflammatory bowel diseases, rheumatoid arthritis, and ankylosing spondylitis [16]. Other studies have demonstrated elevated MPV values in COPD patients [17]. In 2016, Agapakis and coauthors concluded that MPV may be an inflammatory marker and could be a useful tool to detect patients with an increased risk of exacerbation as the mean platelet volume levels were significantly decreased in the exacerbation period in a study carried on adult COPD patients [18].

Proinflammatory cytokines such as CRP, tumor necrosis factor- $\alpha$, and interleukin- 6 are increased in COPD and contribute to the generation of oxidative stress with subsequent platelet activation and also MPV reduction [19]. Moreover, the previous hypothesis is supported by the significantly higher CRP and ESR among the patients' group compared to the normal control group. In our study, the MPV levels had a significant negative correlation with elevated CRP, as the higher CRP values are associated with lower 
Table 3 Comparison between cases and controls; platelets count, MPV, and plateletcrit findings in relation to other parameters

\begin{tabular}{|c|c|c|c|c|}
\hline$N(39)$ & & PLTS & MPV & PCT \\
\hline \multirow[t]{2}{*}{ Age in years } & Correlation coefficient & 0.070 & 0.388 & 0.033 \\
\hline & $P$ value & 0.670 & 0.015 & 0.840 \\
\hline \multirow[t]{2}{*}{ Age of start of illness in years } & Correlation coefficient & 0.050 & 0.346 & 0.032 \\
\hline & $P$ value & 0.764 & 0.031 & 0.846 \\
\hline \multirow[t]{2}{*}{$\mathrm{SO} 2$} & Correlation coefficient & 0.063 & 0.139 & 0.075 \\
\hline & $P$ value & 0.704 & 0.397 & 0.650 \\
\hline \multirow[t]{2}{*}{$\mathrm{HB}(\mathrm{g} / \mathrm{dL})$} & Correlation coefficient & 0.343 & 0.065 & 0.233 \\
\hline & $P$ value & 0.032 & 0.696 & 0.154 \\
\hline \multirow[t]{2}{*}{ TLC } & Correlation coefficient & 0.042 & 0.082 & 0.010 \\
\hline & $P$ value & 0.798 & 0.622 & 0.952 \\
\hline \multirow[t]{2}{*}{ ESR } & Correlation coefficient & 0.158 & 0.168 & 0.062 \\
\hline & $P$ value & 0.336 & 0.307 & 0.705 \\
\hline \multirow[t]{2}{*}{ CRP } & Correlation coefficient & 0.327 & 0.164 & 0.363 \\
\hline & $P$ value & 0.042 & 0.317 & 0.023 \\
\hline
\end{tabular}

MPV, this comes in agreement with the findings of Agapakis et al. [18] However, this finding does not agree with the results obtained by Kalemci et al. [20] were they reported a significant increase in MPV with increasing severity of adult COPD yet, in agreement with the current study they reported an increase in platelets count and subsequently PCT with increased severity of adult COPD.

Plateletcrit represents the actual platelet mass as it constitutes the percentage of the volume that platelets occupy in blood, it normally ranges around $0.2 \%$ blood volume. For such an important role and variable functions to be concentrated in this small volume, this makes the research of variations in platelets number and volume of big value [8].

\section{Conclusion}

To our best knowledge, this is the first study to target the pediatric age group and study the relation of chronic respiratory diseases and simple indices of platelet parameters. Also to our best knowledge, this is the first study of the relation of platelet count, MPV, and PCT with oxygen saturation, fever, change in the pattern of cough, sputum, or dyspnea as indicators of exacerbation; however, the study did not find any significant relation between those mentioned parameters.

More valuable data could be obtained by performing the study on a larger scale, and following up the patients for longer periods to assess the fluctuation in the platelet count and indices in relation to different clinical aspects of the disease.

Table 4 Comparison between cases and controls; platelets count, MPV, and plateletcrit findings in relation to changing pattern of chronic cough and fever

\begin{tabular}{|c|c|c|c|c|c|c|c|c|c|c|c|}
\hline & \multicolumn{10}{|c|}{ Changing pattern of chronic cough, sputum, or dyspnea } & \multirow{3}{*}{$P$ value } \\
\hline & \multicolumn{5}{|l|}{ Yes } & \multicolumn{5}{|l|}{ No } & \\
\hline & Mean & Standard deviation & Median & Minimum & Maximum & Mean & Standard deviation & Median & Minimum & Maximum & \\
\hline PLTS & 405.72 & 192.38 & 344.00 & 154.00 & 819.00 & 363.30 & 130.47 & 370.00 & 193.00 & 523.00 & 0.740 \\
\hline MPV & 8.76 & 0.60 & 8.80 & 7.60 & 10.00 & 8.44 & 0.42 & 8.40 & 7.90 & 9.00 & 0.098 \\
\hline \multirow[t]{4}{*}{ PCT } & 3579.29 & 1798.75 & 3080.00 & 1216.60 & 7698.60 & 5051.04 & 6066.01 & 3474.80 & 1659.80 & 22041.00 & 0.887 \\
\hline & \multicolumn{11}{|l|}{ Fever } \\
\hline & Yes & & & & & No & & & & & $P$ value \\
\hline & Mean & Standard deviation & Median & Minimum & Maximum & Mean & Standard deviation & Median & Minimum & Maximum & \\
\hline PLTS & 420.94 & 217.93 & 328.00 & 168.00 & 819.00 & 374.68 & 141.84 & 400.00 & 154.00 & 600.00 & 0.685 \\
\hline MPV & 8.81 & 0.68 & 9.10 & 7.60 & 10.00 & 8.58 & 0.47 & 8.65 & 7.70 & 9.30 & 0.172 \\
\hline PCT & 3757.55 & 2086.31 & 2853.60 & 1545.60 & 7698.60 & 4110.52 & 4180.63 & 3474.80 & 1216.60 & 22041.00 & 0.878 \\
\hline
\end{tabular}




\section{Abbreviations}

CF: Cystic fibrosis; CLD: Chronic lung disease; CRP: C-reactive protein; ILD: Interstitial lung disease; MPV: Mean platelet volume; PLT: Platelet; PTC: Plateletcrit

\section{Acknowledgements}

Not applicable

\section{Authors' contributions}

ID contributed to the conception and design of the study, collection of data, writing of the manuscript, and final approval of the version to be published. IS shared in putting study design, laboratory work assessment of the studied population, and final approval of the version to be published. EY shared in laboratory work of the study, writing of the manuscript, analysis and interpretation of data, and final approval of the version to be published. All authors read and approved the final manuscript.

\section{Funding}

This research did not receive any specific grant from funding agencies in the public, commercial, or not-for-profit sectors.

\section{Availability of data and materials}

All data generated or analyzed during this study are included in this published article.

\section{Ethics approval and consent to participate}

The study was approved by the Cairo University Ethical Committee ref. number 1-031016. Participation was subject to the verbal informed consent of caregivers.

\section{Consent for publication}

Not applicable.

\section{Competing interests}

The authors declare that they have no competing interests.

\section{Author details}

${ }^{1}$ Pediatrics, Pediatric Pulmonology, Faculty of Medicine, Cairo University, Kasr Alainy, Street, Cairo Post Code: 11562, Egypt. ${ }^{2}$ Clinical and Chemical Pathology, Faculty of Medicine, Cairo University, Kasr Alainy, Street, Cairo Post Code: 11562, Egypt.

Received: 14 November 2019 Accepted: 29 December 2019

Published online: 09 March 2020

\section{References}

1. Baarsma HA, Königshoff M (2017) 'WNT-er is coming': WNT signalling in chronic lung diseases. Thorax 72(8):746-759. https://doi.org/10.1136/ thoraxjnl-2016-209753 Epub Apr 17

2. Niemitz M, Schwerk N, Goldbeck L, Griese M (2018) Development and validation of a health-related quality of life questionnaire for pediatric patients with interstitial lung disease. Pediatric pulmonology 53(7):954-963

3. Qin H, Xu XP, Zou J et al (2019) Krebs von den Lungen-6 associated with chest high-resolution $C T$ score in evaluation severity of patients with interstitial lung disease. Pulmonology 25(3):143-148

4. Lefrançais E, Ortiz-Muñoz G, Caudrillier A et al (2017) The lung is a site of platelet biogenesis and a reservoir for haematopoietic progenitors. Nature 544:7648-7105

5. Weyrich AS, Zimmerman GA (2013) Platelets in lung biology. Annual review of physiology 75:569-591

6. Ulasli SS, Ozyurek BA, Yilmaz EB et al (2012) Mean platelet volume as an inflammatory marker in acute exacerbation of chronic obstructive pulmonary disease. Pol Arch Med Wewn 122:284-290

7. Levent C, Zeynep C (2017) Relationship between coronary tortuosity and plateletcrit coronary tortuosity and plateletcrit. Cardiovasc J Afr 28(6):385

8. Gomez-Casado C, Villaseñor A, Rodriguez-Nogales A et al (2019) Understanding platelets in infectious and allergic lung diseases. Int J Mol Sci 20(7):1730. Published 2019 Apr 8. https://doi.org/10.3390/ijms20071730

9. World Medical Association (2001) World Medical Association Declaration of Helsinki. Ethical principles for medical research involving human subjects. Bull World Health Org 79(4):373
10. Chan YH (2003) Biostatistics102: quantitative data-parametric \& nonparametric tests. Singapore Med J. 44(8):391-396

11. Chan YH (2003) Biostatistics 104: correlational analysis. Singapore Med J. 44(12):614-619

12. Maclay JD, McAllister DA, Johnston S et al (2011) Increased platelet activation in patients with stable and acute exacerbation of COPD. Thorax 66(9):769-774

13. Skoczyński S et al (2019) Chronic Obstructive Pulmonary Disease and Platelet Count. Adv Exp Med Biol Neuroscience and Respiration 43:19-23. https://doi.org/10.1007/5584_2019_379 1-5

14. Martinez-Rivera C, Portillo K, Muñoz-Ferrer A et al (2012) Anemia is a mortality predictor in hospitalized patients for COPD exacerbation. COPD 9(3):243-250

15. Gasparyan Y, Armen LA, Mikhailidis DP et al (2011) Mean platelet volume: a link between thrombosis and inflammation? Curr Pharm Des 17(1):47-58

16. Kisacik B, Tufan A, Kalyoncu U et al (2008) Mean platelet volume (MPV) as an inflammatory marker in ankylosing spondylitis and rheumatoid arthritis. Joint Bone Spine. 75:291-294

17. Bansal R, Gupta HL, Goel A et al (2002) Association of increased platelet volume in patients of chronic obstructive pulmonary disease: clinical implications. J Ind Acad Clin Med. 3:169-172

18. Agapakis DI, Massa EV, Hantzis I, Maraslis S, Alexiou E, Imprialos KP, Damianidou M, Satsoglou E (2016) The role of mean platelet volume in chronic obstructive pulmonary disease exacerbation. Respir Care 61(1):4449. https://doi.org/10.4187/respcare.04132 Epub 2015 Nov 24

19. Wang R-t, Li J-Y, Cao Z-g et al (2013) Mean platelet volume is decreased during an acute exacerbation of chronic obstructive pulmonary disease. Respirology 18(8):1244-1248

20. Kalemci S, Akin F, Sarihan A et al (2018) Relationship between hematological parameters and severity of chronic obstructive pulmonary disease. Pol Arch Intern Med 128:171-177

\section{Publisher's Note}

Springer Nature remains neutral with regard to jurisdictional claims in published maps and institutional affiliations.

\section{Submit your manuscript to a SpringerOpen ${ }^{\circ}$ journal and benefit from:}

- Convenient online submission

- Rigorous peer review

- Open access: articles freely available online

High visibility within the field

- Retaining the copyright to your article

Submit your next manuscript at $\boldsymbol{\nabla}$ springeropen.com 